
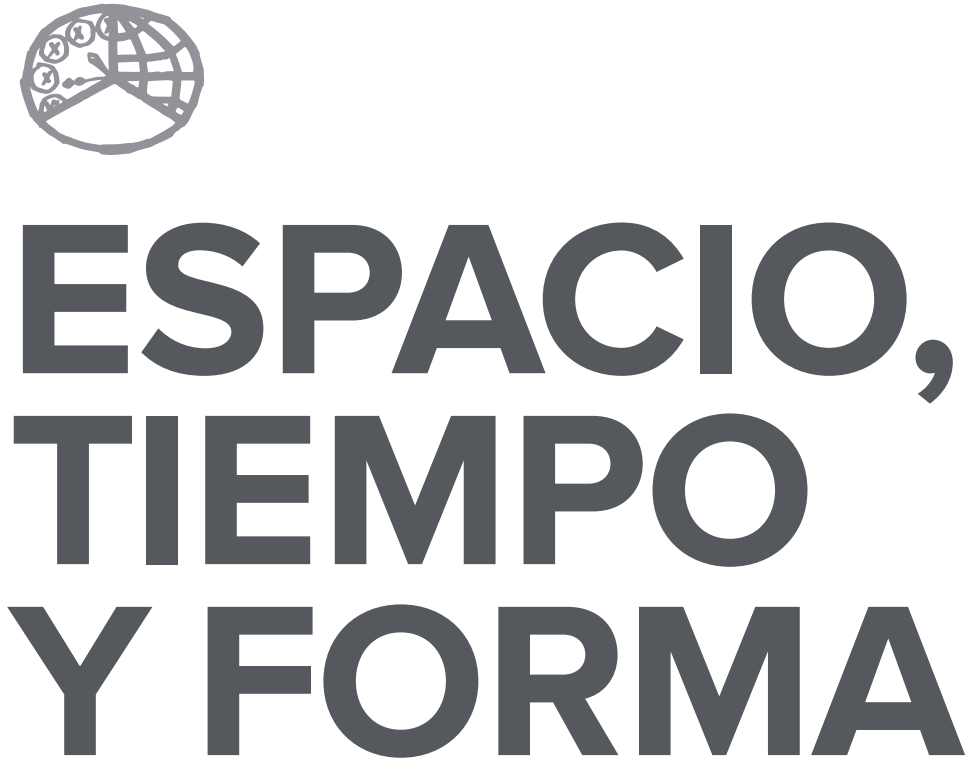

AÑO 2018

ISSN 0214-9745

E-ISSN 2340-1362



SERIE III HISTORIA MEDIEVAL

REVISTA DE LA FACULTAD DE GEOGRAFÍA E HISTORIA 



\section{ARTÍCULOS · ARTICLES}





\title{
RODRIGO DE MENDOZA, I MARQUÉS DEL CENETE Y I CONDE DEL CID: PARALELISMOS ENTRE SU BIOGRAFÍA Y SU PRETENDIDA GENEALOGÍA
}

\section{RODRIGO DE MENDOZA, FIRST MARQUIS OF EL CENETE AND FIRST COUNT OF EL CID: PARALLELS BETWEEN HIS BIOGRAPHY AND HIS ALLEGED GENEALOGY}

\author{
Estefania Ferrer del Río
}

Recepción: 2017/5/3 · Comunicación de observaciones de evaluadores: 2017/9/15 . Aceptación: 2017/9/26

DOI: http://dx.doi.org/10.5944/etfiii.31.2018.18953

\section{Resumen ${ }^{2}$}

El Gran Cardenal de España quiso, al legitimar a sus hijos nacidos de sendas relaciones ilícitas, consolidarlos como miembros de la nobleza castellana. Así fue, por ejemplo, con su primogénito Rodrigo, I marqués del Cenete y I conde del Cid, emparentado por voluntad paterna con Rodrigo Díaz de Vivar, el Cid Campeador. Al apropiarse de esta ficticia genealogía, el prelado dotaba al mayorazgo legado a éste de un simbolismo que, a medida que el marqués fue madurando y curtiéndose en la guerra de Granada, las campañas italianas y las Germanías de Valencia, cobraba mayor verosimilitud a través de sus actos y acciones. El objeto de este trabajo es aproximarnos al perfil biográfico de Rodrigo de Mendoza y reseñar los paralelismos que se observan entre su vida y la que llevó su pretendido y egregio antepasado.

\section{Palabras clave}

Marqués del Cenete; Cid Campeador; paralelismos biográficos; genealogía; siglos XV-XVI.

1. Universitat de València. C.e.: estefaniaferrerdelrio@gmail.com

2. Este artículo se inscribe en el proyecto de investigación «Memoria, imagen y conflicto en el arte y la arquitectura del Renacimiento: la revuelta de las Germanías de Valencia» (HAR2017 - 88707-P), Ministerio de Economía, Industria y Competitividad, Gobierno de España. 


\begin{abstract}
By legitimizing his children born of illicit relations, the Grand Cardinal of Spain wished to make them members of the Castilian nobility. Such was the case, for instance, with his first-born Rodrigo, first marquis of the Cenete and first count of El Cid, allegedly related, through the wishes of his parents, to Rodrigo Diaz de Vivar, El Cid Campeador. By appropriating this fictional genealogy, the prelate endowed his entailed estate (mayorazgo) with a symbolism that earned him greater credibility through his deeds and actions, especially as the marquis matured and gained experience in the war of Granada, the Italian campaign and the Germanías of Valencia. The object of this study is to outline a biographical profile of Rodrigo de Mendoza and establish parallels observed between his life and that of his alleged and eminent ancestor.
\end{abstract}

\title{
Keywords
}

Marquis of El Cenete; Cid Campeador; Biographical Parallels; Genealogy; Fifteenth and Sixteenth Centuries. 


\section{INTRODUCCIÓN ${ }^{3}$}

El Cid Campeador, Rodrigo Díaz de Vivar (¿Vivar del Cid, Burgos?, ca. I048-Valencia, I099) fue un caballero castellano que -tal como relata el Cantar de mio Cid ${ }^{4}$ y es sobradamente conocido desde el siglo XIII- dejó profunda huella en la memoria colectiva desde tiempos de la Reconquista hasta nuestros días. Sus hazañas y servicios tanto a intereses cristianos como musulmanes, sus desencuentros con Alfonso VI y consiguientes destierros, la toma de la ciudad de Valencia y su conversión en señorío hasta iıo2, el peregrinar de sus restos hasta su definitiva ubicación en el crucero de la catedral de Burgos o los casamientos de sus hijas han pervivido en la memoria colectiva desde hace casi un milenio y numerosas estatuas, calles o avenidas esparcidas por las ciudades españolas recuerdan su mítica personalidad.

Sin embargo, las conocidas andanzas del Cid las abordamos en esta ocasión desde una nueva perspectiva, en un contexto como el de la Baja Edad Media en el que está a punto de concluirse la Reconquista con la Guerra de Granada por parte de los Reyes Católicos, a las puertas de la Edad Moderna, al ser Pedro González de Mendoza, cardenal primado de España y consejero áulico de los monarcas, quien trazó meticulosamente los destinos de su hijo primogénito nacido de su relación sentimental con Mencía de Lemos en I468. De hecho, el Cardenal de España, como gustaba titularse por su alta dignidad eclesiástica, aprovechó su paternidad prohibida para bautizar a su vástago como Rodrigo Díaz de Vivar y Mendoza, entroncando de este modo tan particular como inédito con la genealogía del adalid burgalés.

Llegados a este punto, ¿qué motivaciones impulsaron al prelado para relacionar a su descendiente, fruto de una relación ilegítima, con El Cid?, ¿por qué El Cid y no otro personaje legendario?, y dicho esto, ¿cómo educarlo como caballero?, ¿cómo legitimarlo?, ¿con qué patrimonio contaba para poder dotarle de un mayorazgo?, ¿qué dominios eligió para el mismo? En fin, una serie de preguntas que conciernen a la biografía paterna que iremos respondiendo en la medida de lo posible, a las que se añaden otras a lo largo de su intensa vida, desde que en 1489 fuera investido como I conde del Cid $^{5}$ y en I49I como I marqués del Cenete ${ }^{6}, y$

3. Abreviaturas utilizadas: $\mathrm{AGS}=$ Archivo General de Simancas; $\mathrm{AHN}=$ Archivo Histórico Nacional; $\mathrm{C} .=$ caja; $\mathrm{CME}$ = Contaduría Mayor de Hacienda; $\mathrm{D}$. = documento; $\mathrm{f}$. = folio; ff. = folios; leg. = legajo; $\mathrm{p}$. = página; pp. = páginas; $\mathrm{RGS}=$ Registro General del Sello; ss. = siguientes.

4. Para el presente estudio hemos consultado la siguiente edición: Montaner, Alberto (ed.) y Rico, Francisco (ensayo): Cantar de mio Cid. Barcelona, Círculo de Lectores, 2011.

5. Fortaleza que, junto a la de Corlo, fue intercambiada entre Alfonso Carrillo y Pedro González de Mendoza por la villa de Maqueda en 1469, para posteriormente ser cedida a su primogénito en 1489: Pleito homenaje de Rodrigo Albornoz por razón del cambio de la villa de Maqueda por las fortalezas del Cid y del Corlo, hechas entre el obispo de Sigüenza Pedro González de Mendoza y Alfonso Carrillo, 1469, Archivo Histórico Nacional (AHN), Nobleza, Osuna, C. 1708, D. 4-5; Pleito homenaje prestado por Rodrigo de Albornoz sobre entrega de las fortalezas de Maqueda, Cid y Corlo a Alfonso [sic] de Mendoza, Obispo de Sigüenza, 16 de diciembre de 1469, AHN, Nobleza, Osuna, C. 1840, D. 5; Merced de los Reyes Catolicos a Rodrigo de Mendoza, de la fortaleza del Cid, 11 de junio de 1489, AHN, Nobleza, Osuna, D. 1707, D. 7.

6. Mayorazgo de la villas de Cenete, fundado por el cardenal, arzobispo de Toledo a favor de su hijo Rodrigo, 1491, AHN, 
que hicieron de su biografía un cúmulo de coincidencias con las de su presunto antepasado que pretendemos desvelar y dar a conocer.

\section{LAS MOTIVACIONES DEL CARDENAL PEDRO GONZÁLEZ DE MENDOZA}

El obispo Pedro González de Mendoza (I428-I495)7 , sexto hijo del marqués de Santillana, Íñigo López de Mendoza (I398-I458), fue el ejemplo paradigmático del progresivo ascenso de la nobleza castellana afín a la causa isabelina en el conflicto dinástico en forma de guerra civil entablada con su medio hermano Enrique IV 'el Impotente' y -particularmente- con la hija de éste, Juana 'la Beltraneja', pues protagonizó una fulgurante carrera eclesiástica bajo el reinado de Isabel 1 de Castilla y Fernando II de Aragón, los Reyes Católicos, ya que de ejercer como obispo de Calahorra, Sigüenza y Osma, pasó a asumir la dignidad cardenalicia y a ostentar como arzobispo las sedes de Sevilla y Toledo.

Sin embargo, del mismo modo que su contemporáneo Rodrigo de Borja, obispo de Valencia, cardenal y futuro papa Alejandro VI, el cardenal Mendoza no se limitó a ver colmadas sus aspiraciones religiosas y políticas en la jerarquía católica castellana, pues habiendo pasado por alto el voto de castidad sacerdotal también se preocupó por acrecentar sobremanera su patrimonio personal, así como procurar para su descendencia un futuro acorde a su elevada posición social.

En 1458 fallece el marqués de Santillana, en cuyo testamento dejaba a su primogénito Diego Hurtado de Mendoza tanto el mayorazgo como la elección de Ioo libros de su magnífica colección bibliográfica ${ }^{8}$; este hecho nos da a entender dos cuestiones fundamentales: que los bienes del marqués del Cenete (entre ellos, su biblioteca) acumulados por su padre Pedro González de Mendoza no partieron de

\footnotetext{
Nobleza, Osuna, C. 1760, D. 10. Un año más tarde, Rodrigo de Mendoza es facultado por los RRCC para poder crear mayorazgo de sus bienes: Facultad que dieron los Reyes Catolicos a Rodrigo de Mendoza, marqués del Cenete para que hiciese mayorazgo de sus bienes, 3 de octubre de 1492, AHN, Nobleza, Osuna, C. 1858, D. 8; Escritura de mayorazgo con facultad real otorgada por Rodrigo de Mendoza, e incorporaciones de años después, 1492, AHN, Nobleza, Osuna, C. 1760, D. 12-19.

7. Véanse Arteaga y Falguera, Cristina de: La casa del Infantado: cabeza de los Mendoza. Madrid, Duque del Infantado, 1940, vol. I, pp. 170-227; Herrera CASAdo, Antonio: La huella viva del Cardenal Mendoza. Guadalajara, AACHE, 1993; LAYNA SerRano, Francisco: Historia de Guadalajara y sus Mendoza en los siglos XV y XVI. Guadalajara, AACHE, 1993; - Salazar y Mendoza, Pedro de: Crónica de el Gran Cardenal de España, don Pedro Gonçález de Mendoça. Toledo, María Ortiz de Saravia (impr.), 1625.

8. Foulché-Delbosc, Raymond: «Testament du Marquis de Santillane», Revue hispanique: recueil consacréa l'étude des langues, des littératures et de l'histoire des pays castillans, catalans et portugais, 67, 1911, p. 121, recoge la transcripción del testamento original que se halla en el AHN, Nobleza, Osuna, C. 1763, D. 13): «Otrosí mando que.I dicho don Pedro de Mendoça, mi hijo, obispo de Calahorra, aya y se contente en la parte que le pertenesçe aver y heredar de los dichos mis bienes, a rrazón de seys herederos, los lugares de Monasterio y Campillo, que fueron asý mesmo de la dicha mi muger, con sus vasallos y rrentas y pechos y derechos y términos y destritos y terretorios y jurisdiçión cevil y criminal, alta y baxa, mero mixto inperio, y con todo lo de demás pertenesciente al Señorío de los dichos lugares, con lo que.l rruego y mando al dicho mi hijo que se aya por contento de la parte que le pertenesçe aver y heredar de los dichos mis bienes, caso que non sea tanto como le pertenesçiera sueldo por libre, asý por ser Perlado como por otras causas que a ello me mueven [...].»
} 
la herencia de su abuelo el poeta, y que la verdadera herencia que recibió el citado obispo y posterior cardenal y primado fueron los municipios de Monasterio y Campillo (sitos, seguramente, en la provincia de Guadalajara), con la condición de que, dado su estatus eclesiástico, al no tener familia, este patrimonio recibido debía volver al cabeza de familia a su muerte? .

Pero los acontecimientos no se desarrollaron como el propio marqués de Santillana suponía, ya que el cardenal Mendoza tuvo descendencia no legítima sin un título nobiliario que la respaldara ni patrimonio que la sustentara. Así que, dada su influencia con respecto a los Reyes Católicos y su relación con la Santa Sede, consiguió que tanto Isabel ${ }^{\mathrm{Io}}$ como Fernando $^{\text {II }}$, por parte de la Corona de Castilla y de Aragón respectivamente, como también el propio Inocencio $\mathrm{VIII}^{12}$ reconocieran como legítimos a Rodrigo, Diego y Juan con el fin de poder heredar y, por tanto, de poder crear mayorazgo con el patrimonio que el propio cardenal ya había adquirido o adquiriría en adelante.

Es cuando menos curioso que Pedro González de Mendoza, proveniente de una familia con renombre, tuviera quizás en mente emparentar su linaje con El Cid, no sólo por ponerle a su primogénito el nombre de Rodrigo, sino también sus apellidos (anteponiéndolos a los propios) para que no hubiera lugar a dudas; aunque es cierto que todos los genealogistas de los siglos XVI y XVII coincidían en afirmar este hecho: que el linaje de los Mendoza estaba emparentado con Rodrigo Díaz de Vivar, El Cid Campeador ${ }^{13}$.

[...] Ellos Mendozinos que es dicho desde Don Diego Hurtado, Señor de Mendívil, y las casas del Conde de Monteagudo y Castro Morón [...] todos esos traen las armas de la banda verde perfilada dentro en campo de gulas y la cadena de oro y las diez panelas blancas en campo de gulas, pero haber de saber que las panelas son las armas del solar de Mendoza y la banda del linaxe de esos señores Mendoza y Mendozinos y algunos quisieron atribuir al Cid y al linaxe de Bivar. En la Coronica Antigua del Zid, Ruy Díaz, dice que Laín Calvo, Señor de Bivar, ovo en Doña Bello,

9. FoulChÉ-Delbosc, Raymond: op. cit., p. 129: «[...] por cuanto el dicho don Pedro de Mendoça, mi hijo, obispo de Calahorra, non puede aver nin dexar hijos legítimos nin hijas de legítimo matrimonio nasçidos y procreados, mando que los dichos logares que leyo ansý mando que aya y herede por este mi testamento, quiero y dispongo que, después de su vida, los dichos logares los aya y herede y vengan al que al tiempo y sazón subcediere y toviere los dichos mis mayoradgos, segund dicho es [...].»

10. Cédula de la Reina Católica por la que dio licencia y faculto a Pedro González Mendoza, cardenal de España, para hacer mayorazgo de sus bienes a favor de Diego y Rodrigo, hijos de Mencía Lemos y de Juan hijo de Inés de Tobar, legitimados, 12 de mayo de 1487, AHN, Nobleza, Osuna, C. 1858, D. 7.

11. Legitimación de Rodrigo, Diego y Juan de Mendoza, hijos del cardenal de España y arzobispo de Toledo, Pedro González de Mendoza, y Mencía de Lemos, expedida por Fernando el Católico, 21 de mayo de 1489, AHN, Nobleza, Osuna, C. 1760, D. 2.

12. Testimonio (1498) de la bula de Inocencio VIII por la que concedió a Pedro González de Mendoza, cardenal Arzobispo de Toledo y canciller mayor de Castilla, licencia para disponer de los bienes libres en testamento o codicilo, aún a favor de sus hijos si los tuviere, a cuyo efecto les reconocía como si fueran legítimos y en legitimo matrimonio, 1488, AHN, Nobleza, Osuna, C. 1760, D. 1.

13. Catalina García, Juan: «El segundo matrimonio del primer Marqués del Cenete», en VAlera, Juan (ed.): Homenaje a Menéndez Pelayo en el año vigésimo de su profesorado. Madrid, Victoriano Suárez, 1899, pp. 665 y 678; SÁNCHEz CANTÓN, Francisco Javier: La biblioteca del Marqués del Cenete iniciada por el Cardenal Mendoza (1470-1523). Madrid, CSIC, 1942, p. 13; Gómez LoRENTE, Manuel: El marquesado del Cenete (1490-1523). Granada, Universidad de Granada, 1990, p. 60. 
su muger, hixa de Nuño Rasura, abuelo del Conde Fernán Laýnez, y d'este desciende el Zid, Ruy Díaz, y los de Vizcaya [...]. El segundo llevó Laýn Laýnez y d'este descienden los de Mendoza [...] $]^{14}$.

Asimismo, para que este parentesco, seguramente como modo de justificar que su reciente nobleza provenía de legendarios ancestros como El Campeador, tuviera más sentido, además de otros territorios, villas, castillos y marquesados, consiguió por parte de los monarcas el título de conde del Cid para sus posesiones en torno a la fortaleza y villa de Jadraque (Guadalajara) ${ }^{15}$.

Quizá no nos sea de extrañar la justificación que dio de su estirpe el cardenal Mendoza, puesto que, desde los primeros años del siglo XIV, su familia se impuso a la nobleza tradicional, procurando destacar como administradores públicos, fieles servidores o embajadores de los soberanos. Y acabó siendo, sobre todo, en las generaciones posteriores, prácticamente una dinastía a través de ventajosos matrimonios, posibles manipulaciones en las leyes hereditarias y de los servicios a la Corona que proporcionaron a los cabeza de familia sucesivos un incremento tanto en el número de mayorazgos como de villas y territorios bajo su control ${ }^{16}$. El de Rodrigo de Mendoza, es uno de estos ejemplos.

\section{LOS TERRITORIOS ADQUIRIDOS PARA LA FORMACIÓN DEL MAYORAZGO}

Posiblemente, el hecho distintivo entre la familia Mendoza y el resto de las poderosas familias del reino fue saber aprovechar el declive de la vieja nobleza a fines del siglo XIV, proporcionando nuevos líderes políticos y militares en los conflictos que fueron aconteciendo ${ }^{17}$. Su poder se reafirmó y fortaleció gracias a la institución del mayorazgo y la acumulación de dignidades en la persona del cabeza de familia ${ }^{18}$.

Dado que antes de las Cortes de Toro (1505), un hijo no era mayor de edad hasta que su progenitor fallecía, tampoco podía dejar fortuna alguna más allá del círculo familiar ni tenía poder para fundar casa independiente, firmar contrato, mandar un ejército o ensayar actos públicos sin permiso de su padre ${ }^{\mathrm{I} 9}$, el propio cardenal

14. Fernández de Oviedo, Gonzalo: Batallas y quinquaxenas escritas por el Capitán Gonçalo Fernández de Oviedo, criado del Príncipe Don Juan, hijo de los Reyes Cathólicos, y Coronista Mayor de las Indias del Emperador Carlos Quinto, manuscrito, 1601, ff. $337 \mathrm{r}$ y ss.

15. Catalina García, Juan: op. cit., p. 84. La fortaleza de Jadraque fue adquirida en 1469 por el Cardenal Pedro González de Mendoza como fruto de un intercambio con el obispo Alfonso Carrillo de Acuña. Su nomenclatura se debe por aparecer llamada de ese modo en el Cantar de mio Cid.

16. NADER, Helen: The Mendoza Family in the Spanish Renaissance 1350-1550. New Brunswick, Rutgers University Press, 1979, p. 69.

17. Gómez LoRente, Manuel: op. cit., p. 52.

18. Layna Serrano, Francisco y Camarillo Hierro, Tomás: La provincia de Guadalajara. Madrid, Hauser y Menet, 1948, p. 248.

19. NADER, Helen: ibidem, pp. 178-179. 
Mendoza se aseguró de que sus hijos, sobre todo Rodrigo desde $1489,{ }^{20}$ fueran no sólo legitimados, sino también facultados para crear mayorazgo por la reina Isabel.

Conseguida la susodicha legitimación con el fin de que los bienes que concediera a sus hijos no se adscribieran a ninguno de sus obispados -y así formar un verdadero patrimonio para ellos-, el Cardenal de España ya se había preocupado de invertir 20.000.00o de maravedís a través de su contador mayor, Juan de Morales, en comprar villas para que fueran incluidas en las propiedades que estaba acumulando y que luego dividiría entre sus tres hijos ${ }^{21}$.

En concreto, el mayorazgo de Rodrigo de Mendoza fue constituido en Úbeda el 3 de marzo de 1489 con todas aquellas posesiones que el cardenal tenía en el Reino de Castilla: Jadraque y la fortaleza del Cid -además de su tierra-, los sexmos de Henares y Bornoba, Xirueque, Buxalharro, Robredarcas y Matillas (todas ellas ubicadas en la actual provincia de Guadalajara ${ }^{22}$; además de las casas principales que tenía el cardenal en la colación de Santa María de la Fuente y i6o.ooo maravedís en juros ${ }^{23}$. A ello se sumaron las tierras que Pedro González de Mendoza compró en el Reino de Valencia: Alberic, Alcosser, Alasquer ${ }^{24}$, Gavarda ${ }^{25}$ y el castillo de Ayora ${ }^{26}$.

Es el 3 de marzo de I49I, en Guadalajara, cuando le entrega a Rodrigo las siete villas que había obtenido del monarca en tierras reconquistadas, las del marquesado del Cenete: Aldeire, Calahorra, Ferreira, Dólar, Jerez del Marquesado, Lanteira y Alquife, además de otros bienes que, si bien no aparecen mencionados en ninguno de sus mayorazgos, sí llegó a poseer en los términos de las ciudades de Guadix y Granada. Un año más tarde, el ya marqués del Cenete asumió dicho mayorazgo contando con la autorización y el beneplácito reales (el 3 y 5 de octubre de I492, respectivamente). ${ }^{27}$

20. Véase nota 7 .

21. Gómez LORENTE, Manuel: op. cit., p. 137.

22. Idem, p. 138.

23. En las alcabalas, en las rentas del sexmo de Valdearriba, Campo, Pelegrina y la Cabrera (40.000), en las alcabalas y en el sexmo de Bornoba, Henares, Robredarcas y Durón (15.000), en las rentas del Obispado de Sigüenza (35.000) y, por último, en el de Osma y Calahorra (70.000). Franco Silva, Alfonso: «La herencia patrimonial del Gran Cardenal de España D. Pedro González de Mendoza», Historia. Instituciones. Documentos, 9, 1982, pp. 456-46o.

24. Dichas tierras se las cederá a su hijo por vía del mayorazgo creado en Sevilla el 5 de marzo de 1490. GómEZ LORENTE, Manuel: ibidem, p. 138.

25. Giménez Chornet, V., «Població a les baronies d'Alberic, Alcosser, Alasquer i Gavarda als segles XVII i XVIII», en VVAA: Anàlisi local i història comarcal: la ribera del Xúcar (segles XIV-XX). Valencia, Diputació de València, 1990, pp. 143 y ss.; GómeZ-Ferrer, Mercedes: «El Marqués del Zenete y sus posesiones valencianas. Mentalidad arquitectónica y artística de un noble del Renacimiento», Anuario del Departamento de Historia y Teoría del Arte, 22, 2010, pp. 27-46.

26. Lugar adquirido a Diego Gómez de Sandoval por el Cardenal en 1490 como así atestigua la documentación señalada: Capítulos de venta y relación sobre la venta de Ayora (Valencia) entre Diego Gómez de Sandoval y Rodrigo de Mendoza, 1490, AHN, Nobleza, Osuna, C. 1932, D. 24-26; Consentimiento que prestó Diego de Sandoval, hijo del Conde de Castro, a la inversión de los maravedíes que recibió del Cardenal Pedro González de Mendoza y de su hijo Rodrigo por la venta en su favor de Ayora (Valencia), 1490, AHN, Nobleza, Osuna, C. 1933, D. 3. Propiedad cuyo derecho fue ratificado a favor de Rodrigo de Mendoza años después de nuevo por los monarcas: Provisión real recaída en el pleito entre el Duque de Nájera, Pedro Manrique y el Marqués del Cenete, Rodrigo de Vivar de Mendoza sobre derecho a Ayora (Valencia), 3 de octubre de 1500, AHN, Nobleza, Osuna, C. 1932. D. 4; Gómez-Ferrer, Mercedes: op. cit., pp. 34 y ss.

27. Gómez LORENTE, Manuel: ibidem, p. 138. 
Además de todo ello, hay que tener en cuenta el vínculo del deudo que se creó en el contexto de los matrimonios concertados entre la propia aristocracia, actuando, en consecuencia, conjuntamente para reforzar así el cumplimiento de sus intereses, puesto que implicaba una obligación común y un beneficio recíproco ${ }^{28}$ : es el caso del primer matrimonio de Rodrigo, concretado por su padre junto al duque de Medinaceli, con Leonor de la Cerda, unión que le proporcionó una serie de beneficios, posesiones y títulos que, incluso después de su muerte, siguieron reclamando los herederos de la Casa de Medinaceli a los de la Casa del Infantado ${ }^{29}$.

\section{RODRIGO DÍAZ DE VIVAR Y MENDOZA (¿MADRID?, 1468-VALENCIA, 1523)}

El nacimiento de este peculiar condottiero ha generado diversas teorías. Autores como Francisco Javier Sánchez Cantón en su obra La biblioteca del Marqués del Cenete iniciada por el Cardenal Mendoza (I470-I523) aseguran que el nacimiento de Rodrigo de Mendoza debió ser entre I466 y I470 ${ }^{30}$, otros como Manuel Gómez Lorente en su tesis doctoral sobre el marquesado del Cenete opinan que la fecha de su nacimiento no debió darse antes de octubre de $1467^{3 \mathrm{I}}$, o como Miguel Falomir Faus y Fernando Marías ${ }^{32}$, quienes, basándose en el testimonio de la medalla conmemorativa del marqués que se encontraba en el palacio de la Calahorra, defienden que el primogénito del Cardenal de España debió nacer en I473, ya que en la misma, fundida en I499, aparecía una inscripción en la que se decía que el marqués contaba con 26 años de edad. Aun así, en la documentación referente a la curaduría de los hijos de Mencía de Lemos, fechada a 3 de agosto de $1482^{33}$, se asegura que Rodrigo cuenta con I4 años de edad y su hermano Diego con I2, por lo tanto, la fecha de su nacimiento rondaría el año I468.

Rodrigo de Mendoza, como titular del mayorazgo del Cenete, además de otras posesiones en los Reinos de Valencia y de Castilla legadas por su padre, acumuló a lo largo de su vida otros títulos y propiedades merced a sus dos matrimonios

28. Idem, p. 53.

29. Proceso seguido por el Duque de Medinaceli contra los herederos de María de Mendoza, marquesa del Cenete sobre relación de la dote que llevó Leonor de la Cerda al matrimonio con Rodrigo de Mendoza, marqués del Cenete, 1581, AHN, Nobleza, Osuna, C. 1782, D. 8.

30. Sánchez Cantón, Francisco Javier: op. cit., pp. 9-11.

31. Gómez LORENTE, Manuel: op. cit., pp. 82-85.

32. Falomir Faus, Miguel y Marías, Fernando: «El primer viaje a Italia del Marqués de Zenete», Anuario del departamento de Historia y Teoría del Arte, 6, 1994, p. 102.

33. Papeles que se refieren a la curaduría de los hijos de Mencía de Lemos, Rodrigo y Diego de Mendoza (1482), 1481, AHN, Nobleza, Osuna, C. 2028, D. 1. 
con Leonor de la Cerda y Aragón (I493-I497/I498)34 y María de Fonseca y Toledo (I502-I52I $)^{35}$, lo que le convirtieron en uno de los hombres más ricos de su tiempo.

Sin embargo, en muchos de sus actos ${ }^{36}$, el marqués hizo gala durante su vida de un carácter complejo ${ }^{37}$; participó en las guerras de Granada, en las campañas de Italia y en las Germanías de Valencia ${ }^{38}$, encarnando el modelo de hombre instruido en armas ${ }^{39}$, pero, a su vez, iniciado en el coleccionismo al que tan afín eran algunos miembros destacados de su linaje.

Residente en el Reino de Valencia en sus posesiones de Ayora entre I5I2-I4 aproximadamente, en I520-I523 lo encontramos domiciliado en unas dependencias del palacio arzobispal de Valencia, lugar frecuentado por la alta nobleza regnícola ${ }^{40}$, muy probablemente por la inseguridad causada por las acometidas agermanadas y desde donde poder ayudar a su hermano, Diego Hurtado de Mendoza, entonces virrey ${ }^{4}$. Durante esta estancia en la capital del Turia, sabemos que incluso padeció presidio en Xàtiva junto al duque de Calabria ${ }^{42}$, su futuro yerno a título póstumo ${ }^{43}$.

34. Capitulaciones matrimoniales, cartas dote y pago de seguridad de la misma etc, referentes al matrimonio de Rodrigo Mendoza, marqués del Cenete, hijo del Cardenal Arzobispo Pedro González de Mendoza, con Leonor de la Cerda, hija del Duque de Medinaceli, 1492, AHN, Nobleza, Osuna, C. 1782, D. 1-7.

35. El cual le aporta los Señoríos de Coca y Alaejos, a pesar de que Alfonso de Fonseca, poco antes de fallecer la había desheredado. Juro a favor de doña Mayor de Fonseca, ca. 1502, Archivo General de Simancas (AGS), CME, 87, 21. Ferrer del Río, Estefania: «De la bula de Julio II (1504) a las conclusiones de fray Pedro de Álava (1594): el largo proceso de validación del segundo matrimonio de Rodrigo de Mendoza, I marqués del Cenete», Mansucrits: Revista d'Història Moderna, 34, 2016, pp. 13-34.

36. Franco Silva, Alfonso: «La herencia patrimonial...», pp. 453-490; Franco Silva, Alfonso: «Las baronías del Gran Cardenal de España Don Pedro González de Mendoza», en Lluís de Santàngel i el seu temps. València, Ajuntament de València, 1992, pp. 217-227; MARCH, José María: «El primer Marqués del Cenete. Su vida suntuosa», Archivo Español de Arte, 24/93, 1951, pp. 47-51; PONS Fuster, Francisco: «La germanía del notario Joan Sobrevero y los mercaderes», Estudis: Revista de Historia Moderna, 33, 2007, pp. 117-148.

37. Catalina García, Juan: op. cit., pp. 665-681; March, José María: op. cit., pp. 47-51.

38. Duran, Eulàlia (ed.): Cròniques de les Germanies. València, Tres i Quatre, 1984, pp. 14-16.

39. Una educación, centrada, tradicionalmente, en el ejercicio de las armas y la preparación militar y, por tanto, en su formación donde eran valoradas las prácticas que le proporcionan fuerza, agilidad o destreza física, tales como montar a caballo, cazar, ejercitarse con las armas, etc., evolucionará hasta un nuevo tipo de educación en el que, sin olvidar esta preparación física y guerrera, primero primaba el cultivo del espíritu y el ingenio. VARELA, Julia: Modos de educación en la España de la Contrarreforma. Madrid, De la Piqueta, 1983, pp. 26 y ss.; FERRER del Río, Estefania: «La instrucció ideal del príncep humanista a través d'Erasme, Vives i els tractadistes posteriors», Estudis: Revista d'Història Moderna, 41, 2015, pp. 91-112.

40. FerRer VAlLS, Teresa: «Corte virreinal, humanismo y cultura nobiliaria en la Valencia del siglo XVII», en ENCISO, Luis Miguel, y SÁNCHEZ, José Miguel (coords.): Reino y ciudad: Valencia en su historia. Madrid, Caja Madrid Fundación, 2007, pp. 185-200; Gómez-Ferrer, Mercedes: op. cit., pp. 35-37.

41. Diego Hurtado de Mendoza es nombrado virrey y lugarteniente de Valencia en 1520, cargos que ejerce hasta el 1523 en que es sustituido por Germana de Foix: Título de Virrey de Valencia que expidió Carlos I a favor de Diego Hurtado de Mendoza conde de Mélito, 4 de mayo de 1520, AHN, Nobleza, Osuna, C. 1934, D. 1.

42. Arciniega, Luis: El monasterio de San Miguel de los Reyes. Valencia, Biblioteca Valenciana, 2001, vol. I, pp. 166-179; Almenara Sebastià, Miquel y Pardo Molero, Juan Francisco: Borja-Centelles: una polémica familiar en la Valencia del XVI, en LA PARRA, Santiago, y Toldrà, María (eds.): Francesc de Borja, home del Renaixement, sant del Barroc. València, PUV, 2012, pp. 31-32.

43. El emperador Carlos V concreta el matrimonio entre Mencía de Mendoza, viuda desde el 14 de septiembre de 1538, con el viudo de Germana de Foix, el duque de Calabria, en 1541, uniendo así dos grandes casas y, por ende, su extenso y rico patrimonio. 
Así pues, como hemos comentado anteriormente, poco después de establecerse en Valencia con María de Fonseca ${ }^{44}$ y sus tres hijas, Mencía, Catalina y María, acaeció el fallecimiento de su esposa el i6 de agosto de I52I, para lo que el marqués dispuso su sepultura en el monasterio de la Santísima Trinidad de dicha ciudad ${ }^{45}$, concretamente en la capilla contigua al ábside de la iglesia de dicho monasterio, un lugar emblemático, a su vez, por ser el elegido por la reina María de Castilla.

A la desaparición de su esposa se sumaba el cuidado y la educación de sus tres hijas menores de edad en un contexto en el que la revuelta de las Germanías se encontraba en su máxima efervescencia. El apoyo a su hermano intentando frenar el avance de los agermanados llevó a Rodrigo a participar de la captura de Vicent Peris $^{46}$ para su posterior ejecución ${ }^{47}$.

\section{SIMILITUDES ENTRE LAS TRAYECTORIAS DEL CID CAMPEADOR Y DEL I CONDE DEL CID}

Mientras estudios recientes señalan que la estirpe del Cid pertenecía a la alta aristocracia leonesa, la de los Flaínez (o Laínez), señores emparentados con la realeza astur-leonesa por vía paterna a través de Diego Flaínez, acrecentada ésta por parte de su progenitora, proveniente de uno de los linajes de la alta nobleza castellana, el origen de la de nuestro protagonista, Rodrigo de Mendoza, como ya se ha comentado, se hunde en la de los Mendoza oriundos de la localidad alavesa homónima, cuya casa principal fue la de los duques del Infantado. Una familia que, como la del Campeador, estaba estrechamente vinculada a los avatares de su tiempo, marcado por las luchas nobiliarias intestinas, sus servicios a la Corona y el incremento paulatino de sus respectivos patrimonios en el contexto de la Reconquista.

Tal como sucedió con los primeros años del Cid, transcurridos en la corte de Fernando I al servicio del príncipe Sancho, los de Mendoza pasaron en la de los Reyes Católicos junto al príncipe Juan. Dándose la paradoja de que, aunque el

44. Con quien Rodrigo tuvo en su tierna juventud un amor tan apasionado como problemático, para desposarse en secreto en 1502, a pesar de la oposición paterna de María que recurrió a la Reina Católica para que desautorizara dicha unión encarcelando a Rodrigo en las fortalezas de Cabezón y posteriormente de Simancas y a María en la de Zamora antes de ser trasladada al monasterio de las Huelgas en Valladolid. En contra de la voluntad de la propia Isabel I y de la de su familia política, el marqués pudo tener contacto con su esposa, llegando a raptar a María para trasladarse al castillo-palacio de Jadraque.

45. Protocolos del notario Joan Sobrevero, 1521, AHN, Nobleza, Osuna, C. 1342, D. 7-10. SánChez CANTón, Francisco Javier: op. cit., p. 20; Carrasco Martínez, Adolfo: «Los Mendoza y lo sagrado. Piedad y símbolo religioso en la cultura nobiliaria», Cuadernos de Historia Moderna, 25, 2000, p. 238; FerRer del Río, Estefania: «El primer enterramiento del I Marqués del Cenete, Rodrigo Díaz de Vivar y Mendoza, en el convento de la Santísima Trinidad de Valencia», Chronica Nova, 42, 2016, pp. 245-258.

46. Duran, Eulàlia (ed.): op. cit., pp. 14-16.

47. Posiblemente, un acto que celebraría con regocijo después del quebranto ocasionado por las revueltas en sus posesiones valencianas y de su circunstancial encarcelamiento en la prisión real de Xàtiva, coincidiendo, casualmente, con el duque de Calabria, quien, años más tarde, se convertiría, post mortem, en su yerno. 
primero llegó a reinar y aunar en torno a sí las coronas de Castilla, Galicia y León - ejerciendo Díaz de Vivar de alférez real-, pronto murió asesinado en extrañas circunstancias (I072), mientras que el segundo -apadrinado, entre otros, por el Gran Cardenal- no llegó a hacerlo al fallecer en la flor de su juventud después de su boda con Margarita de Austria (I497).

A pesar de que El Cid había combatido a Alfonso VI, sucesor del malogrado Sancho, aquél siguió sirviendo al nuevo monarca hasta su primer destierro (Io8o8I), producido al extralimitarse en su celo guerrero respecto a la taifa de Toledo, bajo amparo del rey castellano. Situación que hizo que el de Vivar tuviera que acudir exiliado al rey de la taifa de Zaragoza a quien prestó sus servicios militares con probado éxito. Hacia I087, los intereses de Alfonso y de Rodrigo vuelven a confluir hasta que en 1088 se produjo el segundo ostracismo del mercenario por las desavenencias acaecidas en la conquista de la taifa de Murcia. Un hecho que, además, conllevó la expropiación de sus bienes y que el militar y sus mesnadas pusieran el punto de mira en la taifa de Valencia, convertida primero en protectorado y conquistada después de sitiada en I098. Lo que hizo que formara parte de su señorío, así como una serie de alianzas políticas y matrimonios entre su parentela y la aristocracia navarra y catalana. A la muerte del Cid en ro99, su mujer, Jimena, apenas pudo retener la ciudad hasta IIo2 gracias a la ayuda de su yerno Ramón Berenguer Ill. En un primer momento, Rodrigo Díaz de Vivar fue enterrado en Valencia, de donde fue trasladado su féretro hasta el monasterio burgalés de San Pedro de Cardeña.

Rodrigo de Mendoza, después de recibir el marquesado del Cenete, sabemos que participó activamente en la última fase de la Guerra de Granada bajo la protección del conde de Tendilla y del adelantado de Cazorla Pedro Hurtado de Mendoza, sus parientes ${ }^{48}$; finalizada ésta con la rendición de la ciudad el 2 de enero de I492, sin apenas un respiro, en I494 se embarcó en Cartagena con destino a Sicilia para servir en las Campañas de Italia comandadas por el Gran Capitán ${ }^{49}$. Previamente, se había desposado con la primogénita del I duque de Medinaceli en abril de I493, matrimonio sin descendencia -por la muerte de su hijo Luis a temprana edad- del que enviudó entre I497-I498. Sabemos que, mientras tanto, el marqués viajó de nuevo a tierras italianas hasta que en 1502 , ya de vuelta, se desposó en secreto con la heredera de Alfonso de Fonseca, señor de Coca y Alaejos, casamiento que provocó su arresto y el de su esposa, María de Fonseca, por parte de los Reyes Católicos, a quienes había irritado la conducta irreductible del hijo primogénito del cardenal Pedro González de Mendoza.

48. Se da la curiosa coincidencia de que el primer episodio bélico en que participó El Cid (la batalla de Alcocer, en Guadalajara), coincide con el nombre de una de las propiedades del marqués en el Reino de Valencia (Alcosser).

49. Hasta el momento se conocían, a través del trabajo de Falomir Faus, Miguel y Marías, Fernando: op. cit., dos viajes del marqués del Cenete a Italia: el primero entre 1499 y 1500 y el segundo entre 1504 y 1506 . Asimismo, quien esto suscribe en colaboración con el Dr. Ferrer Orts, en un trabajo que se encuentra en prensa, demostramos que hubo un viaje anterior a los mencionados, entre 1494 y 1495, en el marco de la I Guerra de Nápoles. 
La muerte de la reina Isabel coincidió con el levantamiento de su prisión, su posicionamiento a favor de Felipe 'El Hermoso' y la marcha de ambos a las posesiones del marqués en Guadalajara (Jadraque), de donde pasaron a La Calahorra, castillo en sus territorios de la vega granadina que remodeló interiormente desde I509 a la romana, es decir, en clave renacentista ${ }^{50}$. Sin embargo, una inscripción en su cortile tenida por ofensiva, seguida de otros alborotos en Granada, causó el malestar en la Corte, ordenándose su eliminación al II conde de Tendilla, a la sazón gobernador de Granada. Circunstancias que, lejos de servir para que sus propietarios habitasen la fortaleza a su gusto, les obligaron a marchar hacia el castillo de Ayora, la más destacada de sus pertenencias en el Reino de Valencia, y la intervención progresiva en sus asuntos entre la alta nobleza regnícola hasta el estallido de la revuelta de las Germanías (1520-23), conflicto bélico en la que participó activamente ya domiciliado en la capital a las órdenes de su hermano Diego, conde de Mélito y virrey de Valencia.

Liberado el marqués de su presidio en Xàtiva y culminada con éxito la derrota de la Germanía, murió en el palacio arzobispal de Valencia en $1523^{51}$, siendo enterrado junto a su esposa en el convento de la Trinidad de la ciudad del Turia. Con posterioridad, su heredera Mencía de Mendoza (casada en primeras nupcias con el conde de Nassau) logró depositar fastuosamente los restos de sus padres en la capilla real del convento de Santo Domingo de Valencia con licencia de Carlos $\mathrm{V}^{52}$, lugar reservado desde antiguo para acoger los despojos de Alfonso «el Magnánimo». Mencía, además, casó en I54I en segundas nupcias con Fernando de Aragón, duque de Calabria y virrey de Valencia, con quien no tuvo descendencia, residiendo en el Palacio del Real de la ciudad y estableciendo una pequeña corte humanista a su alrededor.

50. SCAGLIA, Gustina: «El Codex Escurialensis llevado por el artista a La Calahorra en el otoño de 1509», Archivo Español de Arte, 77/308, 2004, pp. 375-383.

51. Ferrer del Río, Estefania: «El primer enterramiento...», pp. 245-258.

52. García Pérez, Noelia: «Modelos de enterramiento, modelos de patronazgo: la Capilla de los Tres Reyes del Convento de Santo Domingo de Valencia y los Marqueses del Cenete», IMAFRONTE, 19-20, 2007-2008, pp. 63-74, propone que la capilla de los Tres Reyes del convento de Santo Domingo en Valencia había sido la elección de la duquesa de Calabria, Mencía de Mendoza, para su sepultura, junto a sus padres, el marqués del Cenete y María de Fonseca, desde la redacción de su testamento en 1535. Juro a favor de Juan Pardo Tavera (Don Juan de Zúniga), 3 de julio de 1535, AGS, CME, 320, f. 41. Capilla que, a su vez, le fue concedida por el emperador Carlos V el 18 de marzo de 1536, Provisión Real de Carlos I y su madre en la que hacen donación a Mencía de Mendoza, Marquesa de Cenete y Condesa de Nassau, de la Capilla Real del Monasterio de Predicadores de Valencia, 18 de marzo de 1535, AHN, Nobleza, Osuna, C. 1847, D. 6; ya que, originariamente, iba destinada a la sepultura de Alfonso V el Magnánimo (1396-1458) y su esposa María de Castilla (1401-1458), para que también fueran sepultados sus padres y sus futuros descendientes, pero con la condición de que «[...] no se pierda el título de la capilla real y los escudos de armas reales que están en ellas», Provisión Real de Carlos I y su madre en la que hacen donación a Mencía de Mendoza, Marquesa de Cenete y Condesa de Nassau, de la Capilla Real del Monasterio de Predicadores de Valencia, 18 de marzo de 1535, AHN, Nobleza, Osuna, C. 1847, D. 6. 


\section{CONCLUSIONES}

Podemos concluir que los paralelismos entre El Cid y el marqués del Cenete, también I conde del Cid, son más que manifiestos a pesar de no entroncar familiarmente ni coincidir cronológicamente. No cabe duda de que Pedro González de Mendoza, cardenal y consejero de los Reyes Católicos hasta su muerte en I495, ansió para su primogénito ilegítimo una genealogía digna del futuro que había pensado para él. Diríamos que, desde su nombre, no elegido al azar, hasta las posesiones que fue adquiriendo pacientemente en Granada, Guadalajara y Valencia para legárselas en mayorazgo ${ }^{53}$, pasando por su esmerada educación y las campañas militares en las que intervino, algunas de las más destacadas de su tiempo, lograron catapultarlo a las más altas dignidades y responsabilidades políticas de su época.

El carácter altivo e inquieto, indómito diríamos, del marqués le hizo enfrentarse a los propios monarcas a quien debía vasallaje, hasta el punto de llegar a irritarlos y pasar de bando cuando así convenía. Actitud belicosa que, seguramente, le impidió alcanzar las mercedes que para sí obtuvo, por ejemplo, su hermano Diego.

Su destreza militar culminó precisamente en Valencia, la ciudad conquistada por El Cid, en la que ambos murieron a una edad que rondaba los 55 años y fueron enterrados con la pompa debida en algunos de sus edificios más señeros. Hasta su progenie emparentó con la alta nobleza y la realeza, tal cual hizo la del Campeador.

No cabe ningún género de dudas de que en el imaginario del Gran Cardenal y del propio Rodrigo latía la idea de asemejarse lo máximo posible a los avatares biográficos del legendario héroe castellano, pues ambos participaron en fases diferentes de la Reconquista, los dos sirvieron y se enfrentaron a sus reyes por su ímpetu militar y ambición y, finalmente, encontraron en Valencia el escaparate perfecto para alcanzar la gloria. Fama imperecedera la del Cid Campeador a través del Cantar, especialmente aunque no en exclusiva, de la que también participó el marqués gracias al Cancionero General ${ }^{54}$

\section{Vi estar a Don Rodrigo \\ de Mendoça en soledad \\ diciendo solo consigo: \\ «O dama de gran beldad, \\ ¿por qué te has assí comigo?» \\ Mas dezía sin tristura:}

«Dichosa fue mi venturapor darme vos el tormento,

53. Facultad a don Rodrigo de Mendoza, marqués del Cenete, para crear mayorazgo de todos sus bienes y heredades, los cuales detalladamente se expresan, 3 de octubre de 1492, AGS, RGS, Leg. 149210, 2.

54. Perea Rodríguez, Óscar: «Valencia en el Cancionero general de Hernando del Castillo: los poetas y los poemas», Dicenda. Cuadernos de Filología Hispánica, 21, 2003, p. 236, en hace una breve referencia al marqués del Cenete. 
pues a mi conocimiento

no vence sola hermosura,

mas otras gracias sin cuento 55 .

****

Otro Romance he-

cho por Quirós sobre los

amores del Marqués de Cenete con la señora

Fonseca.

Mi desuentura cansada

de los males que hazía

quísome mudar la suerte

por ver si se mandaría

la tristeza y el dolor

que jamás se me partía

por causa de la razón

que a mi muerte se escondía.

Ordenome un pensamiento

de placer y de alegría

que me quitó mis pesares

y diome la fantasía, que si remedio buscasse

ventura me le daría,

si supiesse conocella

que no se me negaría,

y metiome en un desierto

muy solo sin compañía,

adonde caminé tanto

que de mí ya no sabía,

auía tiempo pasado

que de amor me mantenía.

Enderecé mi camino

a vn poblado que ende auía,

do hallé vna fuente seca,

porque el agua que tenía

a quien más la desseava

se le desaparecía.

Yo de sed y de desseo

55. CASTILLO, Hernando del: Cancionero general: que contiene muchas obres de diversos autores antiguos, con algunas cosas nuevas de modernos, de nuevo corregido e impreso. Amberes, Philippo Nucio (impr.), 1573, f. CLXVIr. 
el alma se me salía, si la esperasse o me fuesse qué hazer no me sabía. Vi que jamás pensamiento d.ella no se me partía, reposó sobre razón, pues mudar no me podía. Ya durmiome allí el cuidado que desuelado me auía, y así, de verme durmiendo, vi el agua cómo corría, muy dulce para miralla y amarga a quien la cogía. Mas, de ver mi gloria en ella, de ningún temor temía $y$ allí cargué yo mis ojos hasta que más no podía, y el coraçón y memoria hasta que más no cabía. Mi voluntad ya contenta porque el daño no sabía, díxome: «Señor, despierta despierta, que es ya de día.» $Y$, después que fue despierto, mayores males sentía, que hallé fuente seca más seca que no solía. Mis ojos gastan lo suyo, el coraçón se lo embía, y los dos gastan el cuerpo que.l alma no la tenía.

Que allí se quedó ahogada porque assí lo merecía, si desdicha son amores, júzguese en la vida mía. ****

Mote del Marqués de Cenete.

Quien no te precia, te precia. Glosa de Quirós.

Porque razón lo desprecia, tu valer yo no lo aprecio, 
que, pues, naciste sin precio, quien no te precia, te precia.

$Y$, con este desastre, hallo que mereces tanto que nunca se sabrá quánto ni ay seso para estimarte. Porque.n mí tanto se precia tu valer que no lo aprecio, que, pues, naciste sin precio quien no te precia, te precia ${ }^{56}$.

y a los panegíricos que le dedicaron post mortem los humanistas valencianos del círculo de los duques de Calabria, en particular, las elegías de Joan Àngel González ${ }^{57}$

\section{De Roderico Mendozio Zenetano Marchione Illustrissimo Elegia..$^{8}$}

Ad Iacobum Mendozium Meliti Comitem

Spectatissimum Joannis Angeli Epigramma.

Eloquium Tulli, Fabii mora, dextra Camilli

Restituit cives (Martia Roma) tuos,

Sed tibi plus debet servata Valentia soli.

Quam ducibus Latium (magne Jacobe) tribus;

Dicendo Marcum, Fabium cunctando, Camillum

Pugnando, superas quia tria solus habes,

Unde foro leges: aris sacra, fædera genti.

Restituens positio mante togatus ades,

Quot Tybirina ergo persoluit Roma triumphos,

Illis tot debet Turia, Roma tibi.

Aurea pro nostris igitur ponatur imago,

Quae referat vultus robore cincta tuos.

Talia Iucundo quondam cantavimus ore,

Quum versus ilares læta camæra daret,

Nunctamen ingratas (defuncto fratre) querelas

fundimus et gemitus (magne Jacobe) damus.

Tu tamen hæc legito quamvis sint tristia lectu,

Qux memorant fratris gesta notanda tui.

56. CASTILLO, Hernando del: op. cit., ff. CCXXIVr-v.

57. GonzÁLEZ, Joan Ȧngel: De Roderico Mendozio Zenetano Marchione illustrissimo Elegia. Valencia, Joan Mey (impr.), 1523, ff. 11r-v.

58. Escudo heráldico xilográfico de la familia Mendoza, rodeado de una orla con la inscripción: «Ave Maria, gratia plena.» 
Quo nobis rapto fraternas suscipe partes, In deferta tuos urbs iacet ante pedes.

y Juan Bautista Agnesio ${ }^{59}$.

FRANCISCVS OLIVÆ COMES BAPTI-

STæ SVO, PRO APOLOGETICO ÆDI-

to quondam in laudem Illustrissimi Domini Rhoderici

Zeneti Marchionis, iugulato Vincentio Peres.

Qvod scelus hoc Baptista tuum, dignumque flagellis;

Inuidiæ ne lues, desidiæve, refer.

Alphonsi abscondisse mei præclara potentis

Facta fatis fuerat, carmine digna senis.

Magnanimi cælsas Rhoderici abscondere laudes

Quid iuuat, indignum forte ne laude facis,

Laudaui dices non æqua et laude, tacebis,

Laudandus nullis ergo erat ille modis.

Non stis altiloqui hune laudasset musa Maronis.

Elinguis meritis huius Homerus erit.

Laudasti, laudasse iuuet. Frustra ergo tyranno

Huic victo laudes concinuisse fuit.

Menti Laudaui dices non æqua et laude, tacebis,

Mentix, debes quæ mihi, pende mex.

Quod si doctiloquæ male culta vereris Athenæ

Mittere. Causa isthæc est tibi diffugii.

Cedo libens: sed opus non despuet illa, fidemque.

Nam solet Aoniis docta fauere suis.

Olivæ $\cdot 9 \cdot$ calendas Maii 1542.

59. AgNESIO, Joan Baptista: Apologia in defensionem virorum illustrorum equestrium, bonorumque ciuium Valentinorum: in civilem Valentini populi seditionem [...]. Secunda apologia in laudem [...] Rhoderici Zeneti quondam Marchionis [...]. Apologia in venatores pro auibus [...] cum expositione multarum auium, sermone graeco, latino atque valentino [...]. Valencia, Joan Baldoví y Joan Mey (imprs.), 1543, ff. 31r-v. 


\section{BIBLIOGRAFÍA}

Agnesio, Joan Baptista: Apologia in defensionem virorum illustrorum equestrium, bonorumque ciuium Valentinorum: in civilem Valentini populi seditionem [...]. Secunda apologia in laudem [...] Rhoderici Zeneti quondam Marchionis [...]. Apologia in venatores pro auibus [...] cum expositione multarum auium, sermone graeco, latino atque valentino [...]. Valencia, Joan Baldoví y Joan Mey (imprs.), I543.

Almenara Sebastià, Miquel y Pardo Molero, Juan Francisco: Borja-Centelles: una polémica familiar en la Valencia del XVI, en LA PARRA, Santiago, y ToldrÃ, María (eds.): Francesc de Borja, home del Renaixement, sant del Barroc. València, PUV, 20I2, pp. 25-40.

Arciniega, Luis: El monasterio de San Miguel de los Reyes. Valencia, Biblioteca Valenciana, 200I, 2 vols.

Arteaga y Falguera, Cristina de: La casa del Infantado, cabeza de los Mendoza. Madrid, Duque del Infantado, I940, 2 vols.

Carrasco Martínez, Adolfo: «Los Mendoza y lo sagrado. Piedad y símbolo religioso en la cultura nobiliaria», Cuadernos de Historia Moderna, 25, 2000, pp. 233-269.

CASTILlO, Hernando del: Cancionero general: que contiene muchas obres de diversos autores antiguos, con algunas cosas nuevas de modernos, de nuevo corregido e impreso. Amberes, Philippo Nucio (impr.), I573.

Catalina García, Juan: «El segundo matrimonio del primer Marqués del Cenete», en Valera, Juan (ed.): Homenaje a Menéndez Pelayo en el año vigésimo de su profesorado. Madrid, Victoriano Suárez, I899, pp. 665-68I.

Duran, Eulàlia (ed.): Cròniques de les Germanies. València, Tres i Quatre, I984.

Falomir Faus, Miguel y Marías, Fernando: «El primer viaje a Italia del Marqués de Zenete», Anuario del departamento de Historia y Teoría del Arte, 6, I994, pp. IoI-I20.

Fernández de Oviedo, Gonzalo: Batallas y quinquaxenas escritas por el Capitán Gonçalo Fernández de Oviedo, criado del Príncipe Don Juan, hijo de los Reyes Cathólicos, y Coronista Mayor de las Indias del Emperador Carlos Quinto, manuscrito, I6oI.

FERRER del Río, Estefania: «La instrucció ideal del príncep humanista a través d'Erasme, Vives i els tractadistes posteriors», Estudis: Revista d'Història Moderna, 4I, 20I5, pp. 9I-II2.

FERrer del Río, Estefania: «El primer enterramiento del I Marqués del Cenete, Rodrigo Díaz de Vivar y Mendoza, en el convento de la Santísima Trinidad de Valencia», Chronica Nova, 42, 2016, pp. 245-258.

Ferrer del Río, Estefania: «De la bula de Julio II (I504) a las conclusiones de fray Pedro de Álava (1594): el largo proceso de validación del segundo matrimonio de Rodrigo de Mendoza, I marqués del Cenete», Mansucrits: Revista d'Història Moderna, 34, 2016, pp. I3-34.

FERrER VALLS, Teresa: «Corte virreinal, humanismo y cultura nobiliaria en la Valencia del siglo XVII», en Enciso, Luis Miguel, y SánCHEZ, José Miguel (coords.): Reino y ciudad: Valencia en su historia. Madrid, Caja Madrid Fundación, 2007, pp. I85-200.

Foulché-Delbosc, Raymond: «Testament du Marquis de Santillane», Revue hispanique: recueil consacré à l'étude des langues, des littératures et de l'histoire des pays castillans, catalans et portugais, 67, I9II, pp. II4-I33.

Franco Silva, Alfonso: «La herencia patrimonial del Gran Cardenal de España D. Pedro González de Mendoza», Historia. Instituciones. Documentos, 9, I982, pp. 453-49o.

Franco Silva, Alfonso: «Las baronías del Gran Cardenal de España Don Pedro González de Mendoza», en Lluís de Santàngel i el seu temps. València, Ajuntament de València, I992, pp. 217-227. 
García Pérez, Noelia: «Modelos de enterramiento, modelos de patronazgo: la Capilla de los Tres Reyes del Convento de Santo Domingo de Valencia y los Marqueses del Cenete», IMAFRONTE, I9-20, 2007-2008, pp. 63-74.

GimÉnez Chornet, V., «Població a les baronies d'Alberic, Alcosser, Alasquer i Gavarda als segles XVII i XVIII», en Anàlisi local i història comarcal: la ribera del Xúcar (segles XIV-XX). Valencia, Diputació de València, I990, pp. I43-I55.

GonZÁlez, Joan Àngel: De Roderico Mendozio Zenetano Marchione illustrissimo Elegia. Valencia, Joan Mey (impr.), I523.

Gómez-Ferrer, Mercedes: «El Marqués del Zenete y sus posesiones valencianas. Mentalidad arquitectónica y artística de un noble del Renacimiento», Anuario del Departamento de Historia y Teoría del Arte, 22, 2010, pp. 27-46.

Gómez Lorente, Manuel: El marquesado del Cenete (I490-I523). Granada, Universidad de Granada, I990.

Herrera CaSado, Antonio: La huella viva del Cardenal Mendoza. Guadalajara, AACHE, 1995.

Layna Serrano, Francisco: Historia de Guadalajara y sus Mendoza en los siglos XV y XVI. Guadalajara, AACHE, I993.

Layna Serrano, Francisco y Camarillo Hierro, Tomás: La provincia de Guadalajara. Madrid, Hauser y Menet, I948.

MARCH, José María: «El primer Marqués del Cenete. Su vida suntuosa», Archivo Español de Arte, 24/93, I95I, pp. 47-66.

Montaner, Alberto (ed.) y Rico, Francisco (ensayo): Cantar de mio Cid. Barcelona, Círculo de Lectores, 20II.

NADER, Helen: The Mendoza Family in the Spanish Renaissance I350-I550. New Brunswick, Rutgers University Press, I979.

Perea Rodríguez, Óscar: «Valencia en el Cancionero general de Hernando del Castillo: los poetas y los poemas», Dicenda. Cuadernos de Filología Hispánica, 2I, 2003, pp. 227-25I.

PONS Fuster, Francisco: «La germanía del notario Joan Sobrevero y los mercaderes», Estudis: Revista de Historia Moderna, 33, 2007, pp. II7-I48.

Salazar y Mendoza, Pedro de: Crónica de el Gran Cardenal de España, don Pedro Gonçález de Mendoça. Toledo, María Ortiz de Saravia (impr.), I625.

Sánchez CAntón, Francisco Javier: La biblioteca del Marqués del Cenete iniciada por el Cardenal Mendoza (I470-I523). Madrid, CSIC, I942.

SCAGLIA, Gustina: «El Codex Escurialensis llevado por el artista a La Calahorra en el otoño de I509», Archivo Español de Arte, 77/308, 2004, pp. 375-383.

VARELA, Julia: Modos de educación en la España de la Contrarreforma. Madrid, De la Piqueta, I983. 

Calidad de Revistas

científicas Españolas

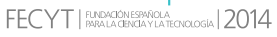

SERIE III HISTORIA MEDIEVAL

REVISTA DE LA FACULTAD DE GEOGRAFÍA E HISTORIA
AÑO 2018

ISSN: 0214-9745

E-ISSN 2340-1362

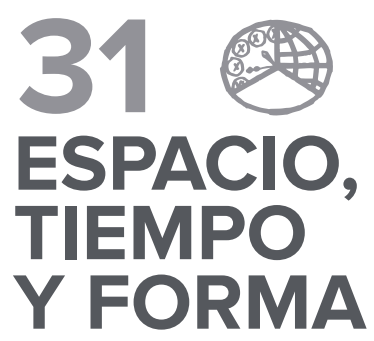

GREGoRIO DEL SER QUIJANO

Carmelo Luis López, In Memoriam

\section{Artículos · Articles}

29 Soha ABBoud Haggar

Un resumen del tratado jurídico de Al-Tafrí: el Manuscrito Árabe 1233 del Monasterio de El Escorial y su supuesta relación con Leyes de Moros

EDUARDo Aznar VALLejo

Norma y conflicto en la navegación castellana bajomedieval

69 Carlos Barquero Goñ

Transferencias de recursos de la Orden de San Juan desde España hasta el Mediterráneo Oriental durante la Edad Media

\section{Margarita Cabrera Sánchez}

La muerte del príncipe Don Juan. Exequias y duelo en Córdoba y Sevilla durante el otoño de 1497

\section{Francisco de Paula Cañas Gálvez}

Primogenitura, continuidad dinástica y legitimitad instituciona en Castilla a principios del siglo XV: Catalina de Trastámara, Princesa de Asturias (1422-†1424)

\section{Paloma Cuenca Muñoz}

El códice visigótico de los Moralia in lob, ms. lat. 83 de la John Rylands Library de Manchester

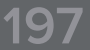

\section{JOSÉ MARÍA DIAGO JIMÉNEZ}

Las instituciones educativas de carácter religioso en el reino hispanovisigodo de los siglos VI y VII a través de los cánones conciliares y las reglas monásticas

MARÍA Díez Yáñez

La Ética aristotélica en Castilla: las bibliotecas universitarias medievales y prerrenacentistas

\section{Estefanía Ferrer del Río}

Rodrigo de Mendoza, I Marqués del Cenete y I Conde del Cid: paralelismos entre su biografía y su pretendida genealogía

\section{ALEJANDRo García Morilla \\ Escritura publicitaria de transición: entre la visigótica y la carolina.} El paradigma burgalés

María Dolores García Oliva

Señores contra campesinos: un conflicto por la tierra en Mirabel a finales de la Edad Media y principios de los Tiempos Modernos (1488-c. 1520)
34.3 jaime García Carpintero lópez de Mota

La hospitalidad santiaguista a finales de la Edad Media: el proyecto de reconstrucción del hospital de Alarcón

377 LAURA DA GRACIA

La posesión agraria individual en los registros notariales de Fuente el Sol (1481-1482

4.03 Mauricio Herrero Jiménez

El valor de los documentos reales en los procesos de la Real Chancillería de Valladolid

431 Miguel josé López-Guadalupe Pallarés

Procesos de señorialización en los concejos de la Extremadura castellano-leonesa. Un estado de la cuestión

455 Ángel Martínez Catalán

Las rentas decimales del cabildo catedralicio de Cuenca a inicios del siglo XV (1400-1432)

4.83 Gonzalo Oliva Manso

Cien años de moneda en Castilla (1172-1268). El siglo del maravedí de oro

521 jesús Olivet García-Dorado

El cabildo de curas y beneficiados de Toledo en la segunda mitad del siglo XV. Composición y aspectos institucionales (1455-1488)

547 Mariel PÉrez

Clérigos rurales, comunidades y formación de las estructuras parroquiales en la diócesis de León (siglos XI-XIII)

575 Milagros Plaza Pedroche

La Orden de Calatrava en la Baja Edad Media (1350-1500): repaso historiográfico

597 Pedro Andrés Porras Arboledas

La pervivencia del Fuero de Cuenca en los inicios de la Modernidad: el testimonio de los fueros de Consuegra y Requena

619 Juan Pablo Rubio Sadia

Los mozárabes frente al rito romano: balance historiográfico de una relación polémica

SANDRA SUÁREZ GARCíA

Los habices de la Vega de Granada como forma de conocimiento del reino nazarí y su transformación tras la conquista: la alquería de La Zubia 


\section{1}

\section{ESPACIO,}

\section{TIEMPO}

\section{Y FORMA}

SERIE III HISTORIA MEDIEVAL

REVISTA DE LA FACULTAD DE GEOGRAFÍA E HISTORIA

\section{De medievalistas ilustres · On Renowned Medievalists}

671 Francisco Abad Nebot

Entradas para un Diccionario

\section{Libros $\cdot$ Books}

679 Almagro Vidal, Clara, Paisajes medievales en el Campo de Calatrava (CARLos BARQUero GoÑI)

681 Carvajal Castro, Álvaro, Bajo la máscara del Regnum. La monarquía asturleonesa en León (854-1037) (JOSÉ MANUEL RODRÍGUEZ GARCíA)

683 Fuente Pérez, María Jesús, Violante de Aragón, reina de Castilla (ANA ECHEVARRía ARsuaga)

687 Martín Prieto, Pablo, Historia del pensamiento medieval: filosofia y teología (FRANCISCO LEÓN FLORIDO)

689 Pardo de Guevara y Valdés, Eduardo (ed.), Mujeres con poder en la Galiciamedieval (siglos XIII-XV). Estudios, biografías y documentos (ENRIQUe CANTERA MONTENEGRO)

693 Ríos Saloma, Martín (ed.), El mundo de los conquistadores (Jessica Ramírez MéndeZ)

690 Solórzano Telechea, Jesús, Arízaga Bolumburu, Beatriz y BOCHACA, Michel (eds.), Las sociedades portuarias de la Europa atlántica en la Edad Media (ANTONIO ORTEGA VILLOSLADA)

703 VAL VALDIVIEso, María Isabel del (coord.), El agua en el
imaginario medieval. Los reinos ibéricos en la Baja Edad Media (MARÍA Jesús Fuente)

707 Villar García, Luis Miguel, Archivo Municipal de Segovia. Documentación medieval, 1166-1474 (ENRIQUE CANTERA MONTENEGRO) 